\title{
A Novel Fuzzy Fusion Algorithm of Multi-sensor Data and Its Application in Coalmine Gas Monitoring
}

\author{
Xiaojie Zhu ${ }^{1,2^{*}}$, Ze Jiang ${ }^{1,2}$, Xiaobing Zhao ${ }^{1,2}$, Mingjie Zhang ${ }^{1,2}$, Xiangfei Chen ${ }^{1,2}$ \\ ${ }^{1}$ CCTEG Changzhou Research Institute, Changzhou 213015, China \\ ${ }^{2}$ Tiandi (Changzhou) Automation Co., Ltd., Changzhou 213015, China
}

Corresponding Author Email: sherylxx@126.com

https://doi.org/10.18280/i2m.180609

Received: 21 April 2019

Accepted: 10 September 2019

\section{Keywords:}

gas monitoring, fuzzy comprehensive evaluation (FCE), multi-sensor data fusion, prewarning

\begin{abstract}
In coalmines, gas disaster is a complex uncertain process influenced by multiple factors. It is difficult to predict gas disaster in an accurate manner. The traditional methods for gas disaster prediction face several common problems, such as the inaccuracy of gas monitoring data, and the unreliable evaluation of gas safety. To solve the problems, this paper proposes a gas monitoring and prewarning method based on fuzzy fusion of multi-sensor data. To realize data fusion and decision-making, the field parameters like wind speed, gas content and temperature, which are monitored by multiple sensors in the coalmine, were allocated to the set of factors, while the decisions on gas state were allocated to the set of remarks. Then, the data collected by the sensors were fuzzified by the fuzzy set theory, creating a fuzzy membership matrix. The fuzzy Cauchy-Riemann equations were introduced to establish the membership function. Furthermore, the coalmine gas state was evaluated and determined in the fusion center under decision-making rules through compositional operation. Based on local decisions, a data fusion decision-making model for coalmine gas disaster was established to make the global decision. The proposed method was applied to analyze the temperature, gas content and wind speed of tunnel face monitored by multiple sensors at three different time points in a coalmine of Shanxi Province, China. The results show that the gas states at all time points were evaluated accurately, without any false or missed alarm, and the prediction based on multi-sensor data fusion was $34 \%$ more accurate than that based on single-sensor data. The research findings provide an effective way to monitor and prewarn the coalmine gas state.
\end{abstract}

\section{INTRODUCTION}

In recent years, coal mining is getting deeper and more intense. More and more coalmines now face the risk of gas outburst. Statistics show that 1,620 coalmines in China have a high gas content, about $29 \%$ of all coalmines in the country. In coalmines, gas hazards mainly include gas outburst and gas explosion. The former refers to the sudden and violent ejection of coal and gas from the coal wall to the mining space. This dynamic disaster is essentially the loss of stability of the coal rock, which contains high-pressure gas, when its energy and damage accumulate to a critical level. This process involves complex nonlinear dynamics [1]. The gas outburst releases a huge amount of energy, causing the roadway to collapse, and brings heavy casualties and property losses. The disaster may also induce secondary incidents like fire and gas explosion $[2$, 3].

According to the National Coal Mine Safety Administration of China, the number of safety accidents and the total number of deaths in coalmines are decreasing steadily from 2006 to 2017; however, gas accidents still take up about $10 \%$ of all coalmine accidents, and the proportion of deaths from gas accidents in the death toll of all coalmine accidents is growing annually [2]. Against this backdrop, it is particularly important to develop an effective prewarning method for gas disaster, which can accurately prewarn the high gas content and risk of gas accidents in accident-prone areas in the coalmine (e.g. the mine face, the tunnel face and the return airway), assess the risk of gas accidents in advance, and thus reduce the probability of gas accidents.

Many scholars and technicians have probed into gas outburst through field observation, theoretical analysis, physical modelling and numerical simulation. Many different views and hypotheses have been developed, concerning the formation mechanism of gas outburst. The multi-factor hypothesis is the most widely accepted explanation of how gas outburst comes into being [3]. The hypothesis holds that gas outburst is the joint result of the deformation potential of the coal and the internal energy of the gas: When the stress state of the coal changes abruptly, the elastic potential accumulated in the coal is suddenly released; then, the coal is broken into fragments, and thrown out under the pressure of the gas. The gas outburst is attributable to the combined effects of multiple factors, including the stress as well as the physical-mechanical properties of the gas and the coal.

Based on the multi-factor hypothesis, three types of gas outburst prediction methods have emerged, namely, the simple index method, sensitivity threshold method and multi-index method. The simple index method performs static discontinuous prediction or dynamic continuous prediction of gas outburst, based on indies like coal quality, gas pressure and gas content. This approach consumes a huge amount of manpower and materials, yet failing to achieve a high safety. The sensitivity threshold method computes the sensitivity of 
each index by the probability theory, determines the initial threshold for each sensitive index based on the computed index dispersion, and then forecasts the gas outburst. The multi-index method introduces various advanced mathematical strategies to gas outburst prediction, such as information fusion, artificial neural network (ANN), fuzzy mathematical theory and comprehensive attribute evaluation. This method can achieve efficient and accuracy prediction of gas outburst [4].

\section{LITERATURE REVIEW}

Recent years has seen much attention being paid to prediction and prewarning of gas disaster. A variety of relevant methods have been developed at scholars at home and abroad [5-10]. An et al. [5] established a set of prewarning criteria for electromagnetic (EM) radiation of gas outburst, after analyzing multiple factors: the features and laws of EM radiation in coal rock damages, as well as the change laws of and correlations between EM radiation and common prewarning indices in gas outburst. Based on the Hadoop platform, Hao and Zhang [6] puts forward a gas outburst prediction and prewarning method in the following steps: the real-time monitored data of gas volume fraction were preprocessed through Holt's exponential smoothing, the characteristic parameters of the preprocessed data were extracted by backpropagation neural network (BPNN), and then the gas outburst prediction and prewarning model was established, coupling the parameters of manual outburst detection. Kursunoglu and Onder [7] screened the prewarning indies for gas outburst, determined the weight of each prewarning index through the analytic hierarchy process (AHP), and constructed a gas outburst prewarning model based on extension theory. Li et al. [8] set up an intelligent discriminant model for gas outburst hazard, which integrates advanced techniques and theories like artificial neural network (ANN), multi-factor pattern recognition, and the theories on mine pressure and gas drainage. Zhang et a. [9] studied the input and output ends of the grey correlation model, using network analysis and multi-class distance discrimination, and then created a multi-index coupling prediction model for gas outburst.

The development of computer technology and information fusion has provided new technical means for gas disaster prediction and prewarning in coalmines. A typical example is multi-sensor data fusion. With the aid of computer technology, this technique fuses the data from multiple sources (sensors) into redundant and complementary information of various homogeneous or heterogeneous sensors. In this way, the monitored object can be evaluated more accurately, laying the basis for correct judgement and decision [10]. Recently, many scholars have successfully applied multi-sensor data fusion in many fields, such as image analysis and processing, robot systems, industrial field parameter detection, and automatic target recognition. The popular ways to fuse multi-sensor data include the neural network (NN), Kalman filtering, Bayesian estimation, and Dempster-Shafer (D-S) theory of evidence [11]. Traditionally, gas outburst is mostly predicted and prewarning based on single-sensor data. Compared with the traditional method, the multi-sensor data fusion boasts many advantages: the real-time data measured by multiple sensors, the data of geological surveys and the empirical data from relevant experts can be fully utilized to identify the key factors affecting the gas state, giving full play to the merits of multisensor configuration.

The multi-sensor data fusion has already been implemented to predict the gas state. For instance, Fan et al. [12] suggested judging the safety level of coalmines by Bayes' theorem and grey correlation analysis. Kuang et al. [13] fused the parameters affecting gas state with the improved BPNN, set up a NN model for gas prediction, and evaluated the gas hazard in a comprehensive manner. Liu et al. [14] combined the NN and the D-S theory of evidence to determine the gas safety of coalmines. Yan et al. [15] collected gas content, ventilation, coal rock dynamics, and parameters of gas disaster (e.g. temperature and coal dust) by multiple types of sensors, integrated the collected data on an expert system, and assigned the confidence to each prediction outcome. Liang et al. [16] analyzed the general framework of multi-sensor data fusion, designed the structure of a gas outburst prewarning system based on multi-sensor data fusion, built up a data fusion model with a feature layer and a decision layer, and realized feedbacks through a multi-sensor management subsystem, achieving closed-loop control of the prediction system. He et al. [17] proposed a systematic gas outburst prewarning model that integrates multi-sensor data, in the light of the hierarchical fusion principle.

The above research methods have greatly enhanced the accuracy of gas state prediction. However, each type of methods has its own limitations: the Bayesian estimation has difficulty in determining the prior probability, the BPNN faces problems like limited learning samples, black box structure and initial weighting of parameters, while the D-S theory of evidence cannot easily yield a reasonable distribution of basic probabilities for specific situations. Therefore, a high-quality gas outburst prewarning system should overcome the defects of different data fusion methods, while making the best use of their respective merits. Through the literature review, it is also learned that multi-sensor data fusion can improve the accuracy and reliability of gas disaster prewarning, and effectively enhance the work safety of coalmines. Of course, there is still ample room to improve the existing methods for gas outburst prediction: the outburst indices should be more diversified, the thresholds should be more objective, and the nonlinearity between indices should be clarified.

Through the above analysis, this paper presents a novel gas outburst prediction method called fuzzy fusion of multi-sensor data, which combines the fuzzy set theory and information fusion technology. In this method, the data collected by multiple types of sensors are subject to comprehensive decision-making by the fuzzy set theory, and feature extraction and fusion; The comprehensive decision-making of the monitored object is achieved through composition operation and the fusion of local decisions from different monitoring points. As such, the proposed method greatly improves the performance of gas monitoring and prewarning system in coalmines.

\section{METHODOLOGY}

\subsection{Fuzzy comprehensive evaluation (FCE) of multi-sensor data}

The FCE is an important evaluation method inspired by the fuzzy set theory. It is a specific application of fuzzy mathematics in risk evaluation. If applied to coalmine gas 
monitoring system, the FCE can make a comprehensive decision of the multi-sensor data.

The basic idea of fuzzy set theory is to make memberships in general sets more flexible, and thus extend the membership of an element for a set to any number in the interval $[0,1]$, rather than 0 or 1 . Hence, the FCE is a suitable method to describe and process the uncertainty of sensor data [16].

Based on multi-sensor data fusion, the comprehensive decision-making can be explained as follows: First, the multisensor data were considered a set of factors $V=\left(v_{1}, v_{2}, \ldots, v_{m}\right)$. Suppose there are $n$ possible gas states judged by the coalmine gas monitoring and prewarning system. Then, the remark set of the decisions can be expressed as $U=\left(u_{1}, u_{2}, \ldots, u_{n}\right)$. During the FCE, the concept of "factor weight vector" was introduced to prevent the decision from being affected by the difference in sensor performance and the signal strength between measuring points. Let $a_{i}=(i=1,2, \ldots, m)$ be the factor weight of each sensor, where $a_{i}$ is a fuzzy subset of the factor set $V$, and $A=\left(a_{1}, a_{2}, \ldots, a_{m}\right)$ be the fuzzy vector. In the coalmine gas monitoring and prewarning system, the factor weight $a_{i}$ that satisfies $\sum_{i=1}^{m} a_{i}=1,\left(a_{i} \geq 0, i=1,2, \ldots, m\right)$ is defined as a factor weight vector. According to the level indices of the remark set, each factor in the factor set $V$ was judged, creating the fuzzy membership matrix:

$$
R=\left\{r_{i j}\right\}_{m \times n}
$$

where, $r_{i j}$ is the membership of $v_{i}$ for $u_{j}$, i.e. the probability to judge state $j$ in $U$ based on a single factor $i$ in $V$.

The probability $B$ of each gas state can be obtained through fuzzy transform. The decision-making of gas state is essentially the composition operation between fuzzy vector $A$ and the fuzzy membership matrix $R$. The two elements were integrated through generalized fuzzy operation, i.e. the larger element is selected in addition, and the smaller element is selected in multiplication. The FCE through fuzzy transform can be expressed as:

$$
B=A \circ R=\left(a_{1}, a_{2}, \cdots, a_{m}\right) \circ\left[\begin{array}{cccc}
r_{11} & r_{12} & \cdots & r_{1 n} \\
r_{21} & r_{22} & \cdots & r_{2 n} \\
\vdots & \vdots & & \vdots \\
r_{m 1} & r_{m 2} & \cdots & r_{m n}
\end{array}\right]=\left(b_{1}, b_{2}, \cdots, b_{n}\right)
$$

where, $b_{j}=\sum_{i=1}^{m} a_{i} r_{i j}, i=1,2, \cdots, n, j=(1,2, \ldots, n)$ is the membership of the correlation between two elements for the state of the $j$-th remark.

\subsection{Structure of multi-sensor data fusion}

The fusion of coalmine gas monitoring data belongs to the decision-level, and targets the local decision at each sensor. First, the data of each sensor were transformed into an independent decision. Then, the decisions from different sensors were integrated in sequence. In other words, the global decision at the fusion center is based on the independent decision at each sensor.

Figure 1 shows the structure of the FCE-based multi-sensor data fusion system. As shown in the figure, each sensor in the system extracts the features from its sensing data, and then complete local decision-making. The local decision is sent to the fusion center, where global decision-making is carried out based on the local decisions.

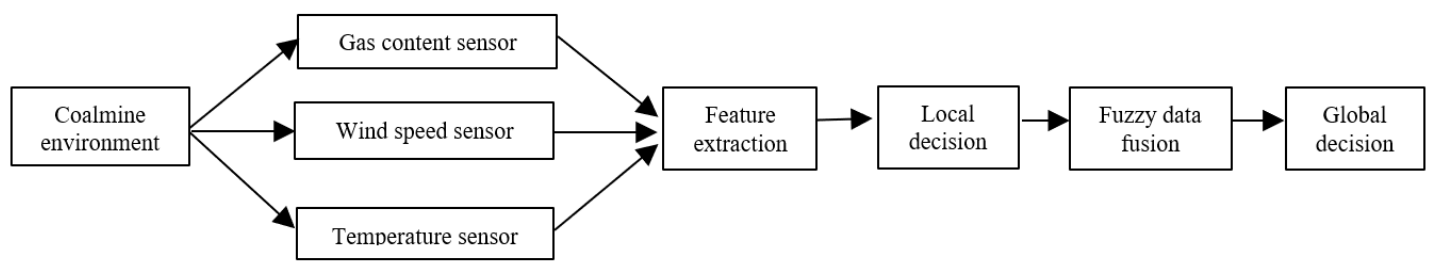

Figure 1. The structure of the FCE-based multi-sensor data fusion system

\subsection{Data fusion algorithm}

In the multi-sensor data fusion system, the $\mathrm{m}$ sensors constitute the factor set $V=\left(v_{1}, v_{2}, \ldots, v_{m}\right)$. The elements in the decision set $D=\left(d_{1}, d_{2}, \ldots, d_{m}\right)$ are generally divided into several levels. The system extends the fusion rules of binary hypothesis testing problem to the case where local sensors are permitted to make a multi-level or soft decision [18].

It is assumed that the sensing domain of each local decision maker can be divided into $j$ nonoverlapping subdomains. If the observation of sensor $i$ lies in the $j$-th subdomain, then $u_{i}=j(j=0,1, \ldots, j-1)$. The local soft decision of each sensor was sent to the fusion center for global decision-making.

Under this fusion structure, the confidence of each level on the decision set $D$ was taken as the decision of each sensor Taking sensor $v_{\mathrm{i}}$ for example, the decision of this sensor $r_{i}=\left(r_{i 1}, r_{i 2}, \ldots, r_{i n}\right)$ was normalized into the input vector to the fusion center $r_{i}^{\prime}=\left(\mathrm{r}_{i 1}^{\prime}, \mathrm{r}_{i 2}^{\prime}, \ldots, \mathrm{r}_{i n}^{\prime}\right)$. Then, the $m \times n$ decision matrix $R$ of vector $r_{i}^{\prime}$ can be established [10]:

$$
R=\left[\begin{array}{cccc}
r_{11}^{\prime} & r_{12}^{\prime} & \cdots & r_{1 n}^{\prime} \\
r_{21}^{\prime} & r_{22}^{\prime} & \cdots & r_{2 n}^{\prime} \\
\vdots & \vdots & & \vdots \\
r_{m 1}^{\prime} & r_{m 2}^{\prime} & \cdots & r_{m n}^{\prime}
\end{array}\right]
$$

Considering the varied roles of the sensors in the data fusion system, different weights (fuzzy subset of $\mathrm{V}$ ) were assigned to different types of sensors, $A=\left(a_{1}, a_{2}, \ldots, a_{m}\right)$, where $a_{i}=\mu\left(v_{i}\right)$ and $i=(1,2, \ldots, m)$ simultaneously satisfy:

$$
\sum_{i=1}^{m} a_{i}=1, \quad a_{i} \geq 0, \quad i=1,2, \ldots, m
$$

The index weights were determined by the AHP. Firstly, the judgment matrix $Q$ was constructed: 


\begin{tabular}{c|cccc}
$q_{k}$ & $q_{1}$ & $q_{2}$ & $\ldots$ & $q_{n}$ \\
\hline$q_{1}$ & $q_{11}$ & $q_{12}$ & $\ldots$ & $q_{1 n}$ \\
$q_{2}$ & $q_{21}$ & $q_{22}$ & & $q_{2 n}$ \\
$\vdots$ & $\vdots$ & $\vdots$ & $\vdots$ & $\vdots$ \\
$q_{n}$ & $q_{n 1}$ & $q_{n 2}$ & $\ldots$ & $q_{n n}$
\end{tabular}

where, $q_{i j}$ is the importance ratio between elements $q_{j}$ and element $q_{j}$ relative to the same superior element $q_{k}$. This ratio was scored against a nine-point scale. Once the judgment matrix $Q=\left(q_{i j}\right)_{\mathrm{n} \times \mathrm{n}}$ is obtained, matrix $A$ was normalized column by column and summed up row by row:

$$
\begin{gathered}
b_{i j}=u_{i j} / \sum_{i=1}^{n} u_{i j} \quad(i, j=1,2, \ldots, n) \\
v_{i}=\sum_{j}^{i} b_{i j} \\
\omega_{i}=v_{i} / \sum_{i=1}^{n} v_{i}
\end{gathered}
$$

After obtaining the approximate value of the feature vector $\omega_{i}$, the accuracy and consistency of the judgment matrix were verified based on the difference between $\lambda_{\max }$ and $n$.

The next step is to determine the membership function of the eigenvalues observed by different sensors relative to each gas state in the coalmine. For this purpose, the gas situation was divided into three fuzzy states: safe $H_{0}$, hazardous $H_{1}$, and extremely hazardous $H_{2}$, and the sensor type and gas state were number as $i=1,2,3$ and $\mathrm{j}=1,2,3$, respectively. Based on the fuzzy Cauchy-Riemann equations, the membership function $\mu_{i j}(x)$ (Figure 2) was defined as:

$$
\begin{gathered}
\mu_{i H_{0}}=\left\{\begin{array}{l}
1, \quad x_{i} \leq a_{i H_{0}} \\
\frac{1}{1+\alpha_{i H_{0}}\left(x_{i}-a_{i H_{0}}\right)^{\beta_{i H_{0}}}}, x_{i}>a_{i H_{0}}, \alpha_{i H_{0}}>0, \beta_{i H_{0}}>0
\end{array}\right. \\
\mu_{i H_{1}}=\frac{1}{1+\alpha_{i H_{1}}\left(x_{i}-a_{i H_{1}}\right)^{\beta_{i H_{1}}}}, \\
\alpha_{i H_{1}}>0, \beta_{i H_{1}} i s \text { positive even number } ; \\
\mu_{i H_{2}}=\left\{\begin{array}{l}
0, \quad x_{i} \leq a_{i H_{2}} \\
\frac{\alpha_{i H_{2}}\left(x_{i}-a_{i H_{2}}\right)^{\beta_{i H_{2}}}}{1+\alpha_{i H_{2}}\left(x_{i}-a_{i H_{2}}\right)^{\beta_{i H_{2}}}}, x_{i} \geq a_{i H_{2}}, \alpha_{i H_{2}}>0, \beta_{i H_{2}}>0
\end{array}\right.
\end{gathered}
$$

where, $\mu_{i H 0}, \mu_{i H 1}$ and $\mu_{i H 2}$ are the memberships of coalmine gas measured by sensor $v_{i}$ for safe, hazardous and extremely hazardous states, respectively; $x_{i}$ is the eigenvalue measured by the sensor measuring system; $a_{i H 0}, a_{i H 1}$ and $a_{i H 2}$ are the standard eigenvalues measured by sensor $i$ for coalmine gas to belong to the three states, respectively; $\alpha_{i H 0}, \alpha_{i H 1}$ and $\alpha_{i H 2}$ are control coefficients (positive constants).

The probability $B$ of each gas state can be expressed as:

$$
B=A \circ R=\left(\mathrm{a}_{1}, \mathrm{a}_{2}, \cdots, \mathrm{a}_{m}\right) \circ\left[\begin{array}{cccc}
r_{11}^{\prime} & r_{12}^{\prime} & \cdots & r_{1 n}^{\prime} \\
r_{21}^{\prime} & r_{22}^{\prime} & \cdots & r_{2 n}^{\prime} \\
\vdots & \vdots & & \vdots \\
r_{m 1}^{\prime} & r_{m 2}^{\prime} & \cdots & r_{m n}^{\prime}
\end{array}\right]=\left(b_{1}, b_{2}, \cdots, b_{n}\right)
$$

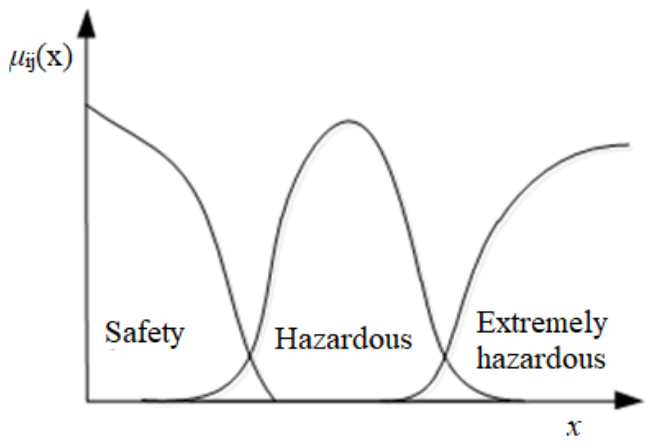

Figure 2. Membership function based on fuzzy CauchyRiemann equations

\section{CASE STUDY AND RESULTS ANALYSIS}

The effective prewarning of gas disaster hinges on the safety assessment of the coalmine. Before making a comprehensive evaluation of the safety in the coalmine, multiple sensors need to be deployed rationally to monitor the field parameters of the mining area and the tunnel face in real time, including temperature, gas content, wind speed and carbon monoxide (CO) content [19-21]. According to the relevant provisions in the Application and Management Standard for Coal Mine Safety Monitoring System and Detector (AQ 1029-2007), the typical sensor layout for the mine face in high-gas content coalmines are shown in Figure 3.

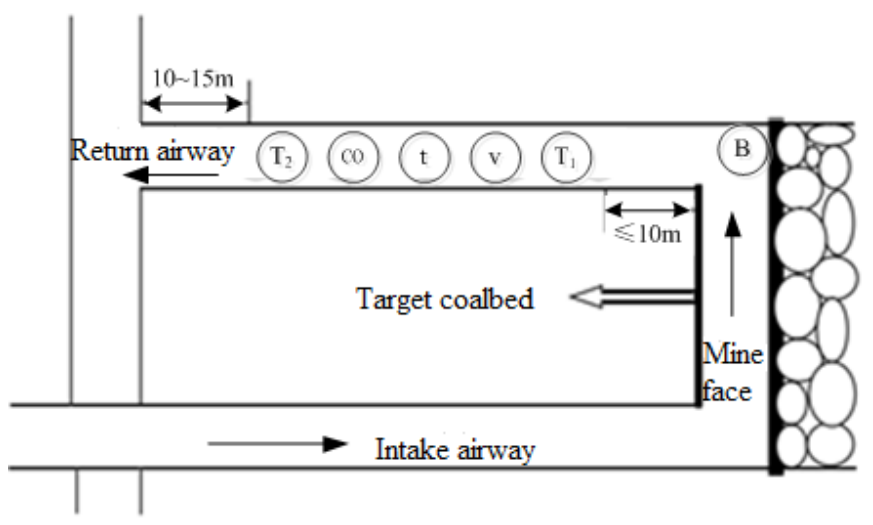

Figure 3. Sensor layout for the mine face

As shown in Figure 3, the intake airway, the mine face and the return airway form a U-shaped ventilation path. A gas detector is deployed on the upper right corner of the path. Gas content sensors are installed on the mine face and the return airway. Other sensors are also provided, including temperature sensor, $\mathrm{CO}$ content sensor and wind speed sensor. In actual applications, each sensor could be replaced with a set of sensors.

Targeting a coalmine in Shanxi Province, China, our analysis mainly focuses on three field parameters of the mine face and the tunnel face, namely, temperature, gas content and wind speed. The data from temperature sensor, wind speed 
sensor and gas content sensor were collected to analyze the gas state of the coalmine. According to the Coal Mine Safety Rules and the intervals of characteristic parameters, the gas situation of the coalmine was divided into such three levels as safe, hazardous and extremely hazardous.

Therefore, the sensor set can be expressed as $V=\left(v_{1}, v_{2}, v_{3}\right)=\{$ temperature sensor, wind speed sensor, gas content sensor\}, and the gas state set can be expressed as $U=\left(u_{1}, u_{2}, u_{3}\right)=\{$ safe, hazardous, extremely hazardous $\}$. The weights were assigned empirically as $A=\left(a_{1}, a_{2}, a_{3}\right)=(0.106$, $0.260,0.634)$. According to the survey data on the coalmine, three sets of data (Table 1), which were collected from a representative tunnel face at different time, were selected for analysis.

Based on the received sample data, the fusion center makes local decisions for the data from each sensor, and determines the monitored state through normalization. Then, the normalized data formed a judgement matrix and the composition operation was performed. Finally, the global decision was made by the maximum membership method. The comprehensive evaluation results of the gas state are listed in Tables 2-4.

Table 1. The data collected by different sensors from the tunnel face

\begin{tabular}{cccc}
\hline Time & $\boldsymbol{t}_{\mathbf{1}}$ & $\boldsymbol{t}_{\mathbf{2}}$ & $\boldsymbol{t}_{\mathbf{3}}$ \\
\hline Temperature ${ }^{\circ} \mathrm{C}$ & 20.3 & 23.2 & 23.8 \\
Wind speed $\mathrm{m} / \mathrm{s}$ & 1.45 & 2.9 & 2.78 \\
Gas content $\%$ & 0.24 & 0.76 & 2.65 \\
\hline
\end{tabular}

Table 2. Fusion results of multi-sensor data collected at time $t_{1}$

\begin{tabular}{ccccc}
\hline State & Safe & Hazardous & Extremely hazardous & Result \\
\hline Temperature sensor $v_{1}$ & 0.538 & 0.461 & 0 & Unknown \\
Wind speed sensor $v_{2}$ & 0.832 & 0.183 & 0 & Safe \\
Gas content sensor $v_{3}$ & 0.558 & 0.439 & 0 & Safe \\
Fusion result & 0.630 & 0.368 & 0 & Safe \\
\hline
\end{tabular}

Table 3. Fusion results of multi-sensor data collected at time $t_{2}$

\begin{tabular}{ccccc}
\hline State & Safe & Hazardous & Extremely hazardous & Result \\
\hline Temperature sensor $v_{1}$ & 0.129 & 0.871 & 0 & Hazardous \\
Wind speed sensor $v_{2}$ & 0.390 & 0.608 & 0 & Hazardous \\
Gas content sensor $v_{3}$ & 0.461 & 0.537 & 0 & Unknown \\
Fusion result & 0.412 & 0.613 & 0 & Hazardous \\
\hline
\end{tabular}

Table 4. Fusion results of multi-sensor data collected at time $t_{3}$

\begin{tabular}{ccccc}
\hline State & Safe & Hazardous & Extremely hazardous & Result \\
\hline Temperature sensor $v_{1}$ & 0.029 & 0.106 & 0.858 & Hazardous \\
Wind speed sensor $v_{2}$ & 0.542 & 0.471 & 0 & Unknown \\
Gas content sensor $v_{3}$ & 0.128 & 0.179 & 0.692 & Extremely hazardous \\
Fusion result & 0.231 & 0.252 & 0.534 & Extremely hazardous \\
\hline
\end{tabular}

The experimental results show that, when the evaluation was based on the data collected by a single sensor, the gas state was sometimes unknown, i.e. it was impossible to tell if the gas state is safe or hazardous. After the fuzzy fusion of multisensor data, it is confirmed that the gas state was safe, hazardous and extremely hazardous at $\mathrm{t}_{1}, \mathrm{t}_{2}$ and $\mathrm{t}_{3}$, respectively. The prediction based on multi-sensor data fusion was $34 \%$ more accurate than that based on single-sensor data. Compared with that based on single-sensor data, the membership based on fuzzy fusion of multi-sensor data gives full play to the merits of information fusion, enhances the accuracy of coalmine gas monitoring, reduces the probability of false alarm or missed alarm, mitigates the uncertainty of gas state evaluation, and improves the performance of the prewarning system.

\section{CONCLUSIONS}

The traditional methods for gas disaster prediction face several common problems, such as the inaccuracy of gas monitoring data, and the unreliable evaluation of gas safety. Moreover, the gas state evaluation based on single-sensor data often has a poor accuracy, which affects the reliability and stability of the prewarning system. Therefore, this paper fully combines the FCE and information fusion technology, and proposes a gas monitoring and prewarning method based on fuzzy fusion of multi-sensor data. The main conclusions are as follows:

(1) Multiple sensors were adopted to monitor the field parameters of a coalmine in real time, including wind speed, gas content and temperature. The parameters were treated as the factors for decision-making based on fused data. The data collected by the sensors were fuzzified, a fuzzy membership matrix was constructed, and the membership function was set up based on fuzzy Cauchy-Riemann equations. Taking the decisions on gas state as the remarks, the authors created a fuzzy evaluation model for gas state, which can predict gas disaster accurately and reliably. In this way, the work safety of the coalmine can be improved effectively.

(2) Under the general framework of multi-sensor data fusion, the coalmine gas state was evaluated and determined in the fusion center under decision-making rules through compositional operation. Based on local decisions, a data fusion decision-making model for coalmine gas disaster was established to make the global decision. The proposed method 
was applied to analyze the data monitored by multiple sensors in a coalmine of Shanxi Province, China. The results show that the prediction based on multi-sensor data fusion was $34 \%$ more accurate than that based on single-sensor data, laying the basis for real-time decision-making.

(3) The proposed method achieves fuzzy fusion of multisensor data in coalmines, and provides an accurate way to estimate and determine the gas state. With the aid of our method, the operators can take proper measures in time to prevent gas disasters.

\section{ACKNOWLEDGEMENT}

This research is supported by the Special Support Project of Science and Technology Innovation and Entrepreneurship Fund of Tiandi Technology Co., Ltd. (Grant No.: 2018-TDQN018).

\section{REFERENCES}

[1] Zang, J., Chen, L.Q. (2017). Complex dynamics of a harmonically excited structure coupled with a nonlinear energy sink. Acta Mechanica Sinica, 33(4): 801-822. https://doi.org/10.1007/s10409-017-0671-x

[2] Black, D.J. (2019). Review of coal and gas outburst in Australian underground coal mines. International Journal of Mining Science and Technology, 29(6): 815-824. https://doi.org/10.1016/j.ijmst.2019.01.007

[3] Qi, W.S., Ling, B.C., Cai, S.J. (2013). Developing Trendand Perspective in the Research of Predicting the Coaland Gas Outburst. China Safety Science Journal, 23(4): 45-50.

[4] Zhu, Z.J., Zhang, H.W., Han, J., Song, W.H. (2013). Prediction of Coal and Gas Outburst Based on PCA-BP Neural Network. China Safety Science Journal, 4.

[5] An, F.H., Yuan, Y., Chen, X.J., Li, Z.Q., Li, L.Y. (2019). Expansion energy of coal gas for the initiation of coal and gas outbursts. Fuel, 235(1): 551-557. https://doi.org/10.1016/j.fuel.2018.07.132

[6] Hao, T.X., Zhang, C.L. (2017). Study on Hadoop platform based method for gas outburst prediction and early warning. China Safety Science Journal, 27(11): 6166.

[7] Kursunoglu, N., Onder, M. (2019). Application of structural equation modeling to evaluate coal and gas outbursts. Tunnelling and Underground Space Technology, 88(6): 63-72. https://doi.org/10.1016/j.tust.2019.02.017

[8] Li, S., Luo, M.K., Fan, C.J., Zhang, S., Bi, H.J. (2016). Research on coal and gas outburst risk intelligent recognition in mining face. China Safety Science Journal, 26(10): 76-81.

[9] Zhang, Y.Y., Cui, J.L., Jiao, X.D. (2018). Study of the multi-index coupling forecasting model of coal and gas outburst and its application. Chinese Journal of Engineering, 40(11): 1309-1316.

[10] Chair Z, Varshney P K. (1986). Optimal data fusion in multiple sensor detection systems. IEEE Trans, AES 22(1): 98-101. https://doi.org/10.1109/TAES.1986.310699

[11] Grychowski, T. (2014). Multi sensor fire hazard monitoring in underground coal mine based on fuzzy inference system. Journal of Intelligent \& Fuzzy Systems, 26(1): 345-351. https://doi.org/10.3233/IFS-120743

[12] Fan, C.J., Li, S., Luo, M.K., Du, W.Z., Yang, Z.H. (2017). Coal and gas outburst dynamic system. International Journal of Mining Science and Technology, 27(1): 49-55. https://doi.org/10.1016/j.ijmst.2016.11.003

[13] Kuang, L., Zhao, W.Q., Yu, Y. (2018). Research on the Prediction Model and Case of Coal and Gas Outburst in Tunnel by Using BP Neural Network. Journal of Railway Engineering Society, 35(2): 56-61.

[14] Liu, H.B., Li, Y.B., Wang, F.Z. (2016). Evaluation Strategy of Gas Outburst Based on Fuzzy Neural NetWork and Evidence Theory. University of Shanghai for Science and Technology, 38(2): 168-171. https://doi.org/10.13255/j.cnki.jusst.2016.02.012

[15] Yan, Z.G., Yao, K., Yang, Y.X. (2017). A novel adaptive differential evolution SVM model for predicting coal and gas outbursts. Journal of Difference Equations and Applications, 23(1): 238-248. https://doi.org/10.1080/10236198.2016.1214725

[16] Liang, Y.Q., Guo, D.Y., Huang, Z.F., Jiang, X.H. (2017). Prediction model for coal-gas outburst using the genetic projection pursuit method. International Journal of Oil Gas and Coal Technology, 16(3): 271-282. https://doi.org/10.1504/IJOGCT.2017.087042

[17] He, C.M., Liu, X.R., Li, J. Wang, Z.J. (2014). Risk analysis of gas outburst tunnel construction based on the fuzzy comprehensive evaluation method. Electronic Journal of Geotechnical Engineering, 19(2014): 86438654.

[18] Wang, H.J., Zhang, Q. (2019). Dynamic identification of coal-rock interface based on adaptive weight optimization and multi-sensor information fusion. Information Fusion, 51: 114-128. https://doi.org/10.1016/j.inffus.2018.09.007

[19] Tang, Y.B. (2017). Experiments simulation of coal and gas outbursts and the factors influencing the outburst process. World of Mining-Surface and Underground, 69(1): 36-40.

[20] Feng, Z.Y., Hu, R., Liu, Q.L. (2013). A method for gas outburst volume detection based on multi-sensor information fusion in the coal mine. Journal of Information and Computational Science, 10(18): 61216130.

[21] Yang, Y.G., Mu, Y.L., Qin, H.Y. (2018). Research on time series characteristics of gas concentration at working face and application of them to early warning. China Safety Science Journal, 28(3): 120-125. 